\title{
Domain of validity and fit of Gent-Thomas and Flory-Erman rubber models to data
}

\author{
T. Beda ${ }^{*}, H$. Gacem ${ }^{2}$, Y. Chevalier ${ }^{2}$, P. Mbarga ${ }^{1}$ \\ ${ }^{1}$ Department of Industrial and Mechanical Engineering, Ecole Nationale Supérieure Polytechnique, University of \\ Yaounde I, BP 8390 Yaounde, Cameroon \\ 2Institut Supérieur de Mécanique de Paris, Laboratoire d'Ingénierie des Structures Mécaniques et des Matériaux \\ (LISMMA), Supméca de Paris. 3, rue F. Hainaut, 93407 Saint-Ouen Cedex, France
}

Received 13 May 2008; accepted in revised form 10 July 2008

\begin{abstract}
In rubber investigation, many strain energy models have been proposed for the hyperelastic behavior. One of earlier models, that of Gent-Thomas (1958), based on theoretical and experimental considerations, has been forsaken in the rubber modeling literature, because of difficulties in establishing its domain of validity and then evaluating the relative mechanical parameters. This note presents how to validate a partial model that is suitable just in a portion of the complete experimental domain: moderate, transition and stiffening deformations. Illustrations of domain validation for the incomplete phenomenological Gent-Thomas model and a simulation with the constrained chain model of Flory-Erman are exposed and their numerical characterisation from experimental data is also revealed.
\end{abstract}

Keywords: rubber, partial modeling, domain validation, mechanical properties, identification process

\section{Introduction}

In behavior modeling of hyperelastic materials, the stress-stretch relations are obtained from the strain energy function. The problem is the mastery of this strain energy function $W$. In material investigation, many starting forms of this energy have been proposed [1-5]. The use of an energy form depends on the mastery of parameters estimation from experimental acquisitions (or from molecular considerations). A principal reason why some earlier interesting models such as that of Gent-Thomas [2] were not often used, lies in the fact that the common procedure (least squares in single process) of parameter estimation does not allow their identification within a restricted region of the experimental (complete) domain. However, the earlier practical Mooney (or Mooney-Rivlin) model [1], which unfortunately fails in predicting other deformation modes such as shear or biaxial deformation, is frequently used (even recently [6]), in hyperelastic literature because its validation and fitting are well mastered by the use of the Mooney plot technique (the plot of the reduced stress as a function of reciprocal stretch in extension-compression). Let us note that according to Mooney model, the secant shear modulus is independent of the stretch, i.e., is constant. This is incorrect since experiment shows that the reduced shear stress varies as the stretch varies $[7,8]$.

The fit of a model to data is an essential means for validating a model in hyperelasticity $[8,9]$.

\section{Hyperelastic uniaxial behavior}

The uniaxial behavior of incompressible, homogenous and isotropic Green materials is given by Equation (1): 
$\sigma_{n}=\frac{\partial W}{\partial \lambda}$

where $W$ is the strain energy function, $\sigma_{n}$ the nominal stress and $\lambda$ the stretch. This law can be rewritten in the strain invariants-based by Equation (2):

$\sigma_{n}(\lambda)=2\left(\lambda-\frac{1}{\lambda^{2}}\right)\left(\frac{\partial W}{\partial I_{1}}+\frac{1}{\lambda} \frac{\partial W}{\partial I_{2}}\right)$

where $I_{1}=\lambda^{2}+2 \lambda^{-1}$ and $I_{2}=2 \lambda+2 \lambda-2$ are respectively the first and the second strain invariants.

The reduced stress $\phi(\lambda)$ is defined by Equation (3):

$$
\phi(\lambda)=\frac{\sigma_{n}}{\left(\lambda-\lambda^{-2}\right)}=\frac{\sigma}{\left(\lambda^{2}-\lambda^{-1}\right)}
$$

where $\sigma$ is the stress (Cauchy stress).

\section{Validating domain and fitting the phenomenological Gent-Thomas model}

The choice of the continuum Gent-Thomas model [2] lies in the fact that this model verifies theoretical and experimental requirements as concerns the dependence of the energy strain function on the strain invariants $I_{1}, I_{2}$; and conditions on $\partial W / \partial I_{1}$ and $\partial W / \partial I_{2}[10,11]$. The Gent-Thomas model is given by Equation (4):

$W_{G T}=K_{1}\left(I_{1}-3\right)+K_{2} \ln \frac{I_{2}}{3}$

where $K_{1}$ and $K_{2}$ are material characteristics. The relative uniaxial behavior law can be rewritten, from Equations (2) and (3), as in Equation (5):

$\phi_{G T}=2\left(K_{1}+K_{2} \frac{\lambda}{2 \lambda^{3}+1}\right)$

where $\phi_{G T}$ is the reduced stress according to the Gent-Thomas model.

\subsection{Ordinary procedure for parameters identification}

The ordinary least squares procedure considers as base of approximation $\langle P(\lambda)\rangle$ given by Equation (6): $\langle P(\lambda)\rangle=\left\langle\xi_{1}(\lambda)=1, \xi_{2}(\lambda)=\frac{\lambda}{2 \lambda^{3}+1}\right\rangle$

$\xi_{k}(\lambda)$ is a generating function. This leads to the estimated parameters $K_{1}$ and $K_{2}$ given by Equation (7):

$\left\{\begin{array}{l}K_{1} \\ K_{2}\end{array}\right\}=\left[\begin{array}{ll}L_{11} & L_{12} \\ L_{12} & L_{22}\end{array}\right]^{-1}\left\{\begin{array}{l}f_{1} \\ f_{2}\end{array}\right\}$

The matrix coefficients are given by Equations (8) and (9):

$$
\begin{aligned}
L_{i j} & =\sum_{k=1}^{M} \xi_{i}\left(\lambda_{k}\right) \xi_{j}\left(\lambda_{k}\right) \\
f_{i} & =\sum_{k=1}^{M} \xi_{i}\left(\lambda_{k}\right) \phi\left(\lambda_{k}\right)
\end{aligned}
$$

Thus, Equations (10), (11), (12), (13) and (14) give the values of the coefficients:

$L_{11}=M$

$L_{12}=\sum_{k=1}^{M} \frac{\lambda_{k}}{2 \lambda_{k}^{3}+1}$

$L_{22}=\sum_{k=1}^{M} \frac{\lambda_{k}^{2}}{\left(2 \lambda_{k}^{3}+1\right)^{2}}$

$f_{1}=\sum_{k=1}^{M} \frac{\sigma_{n k}}{\lambda_{k}-\lambda_{k}^{-2}}$

$f_{2}=\sum_{k=1}^{M} \frac{\lambda_{k} \sigma_{n k}}{\left(2 \lambda_{k}^{3}+1\right)\left(\lambda_{k}-\lambda_{k}^{-2}\right)}$

where $\left(\lambda_{k}, \sigma_{n k}\right)$ are experimental data.

The estimated parameters are recorded in Table 1.

Table 1. Estimated parameters of the Gent-Thomas model from Pak-Flory elastomer data

\begin{tabular}{|l|c|c|}
\hline \multicolumn{1}{|c|}{ Parameters } & $\mathbf{K}_{\mathbf{1}}$ & $\mathbf{K}_{\mathbf{2}}$ \\
\hline Simple Process (SP) & 0.0707 & 0.1321 \\
\hline Multi-Stage Process (MSP) & 0.0671 & 0.1456 \\
\hline
\end{tabular}

\subsection{Multi-stage procedure}

Let us consider, for $x \in V_{N}, \xi_{N}(x)$ being the equivalent function of the function $y(x)$, this leads to Equation (15): 
$y(x) \approx a_{N} \xi_{N}(x)$ for $x \in V_{\Lambda}$

Plotting $y(x)$ versus $\xi_{N}(x)$, that is, $\left(\xi_{N}(x), y(x)\right)$, should give a linear curve for $\mathrm{x} \in V_{N}$. The method consists in plotting the curve $\left(\xi_{k}(x), y(x)\right)$ by varying $k$ to get a linear portion for certain values of $x$, which defines the identification sub-domain. The partial solution $\xi_{k}(x)$ is then determined and the weighting coefficient $a_{k}$ is given by the slope of the relative linear curve. Reiterating this procedure leads to the following recurrence formula [12] given by Equation (16):

$$
\begin{aligned}
& y_{k}(x)=y(x)-\sum_{j=0}^{k-1} a_{N-j} \xi_{N-j}(x) ; k=1, N \\
& y_{0}(x)=y(x)
\end{aligned}
$$

where $y_{k}(x)$ is the function to be identified in the sub-domain $V_{k}$.

To evaluate the Gent-Thomas parameters, one has first to look for the valid domain of the model.
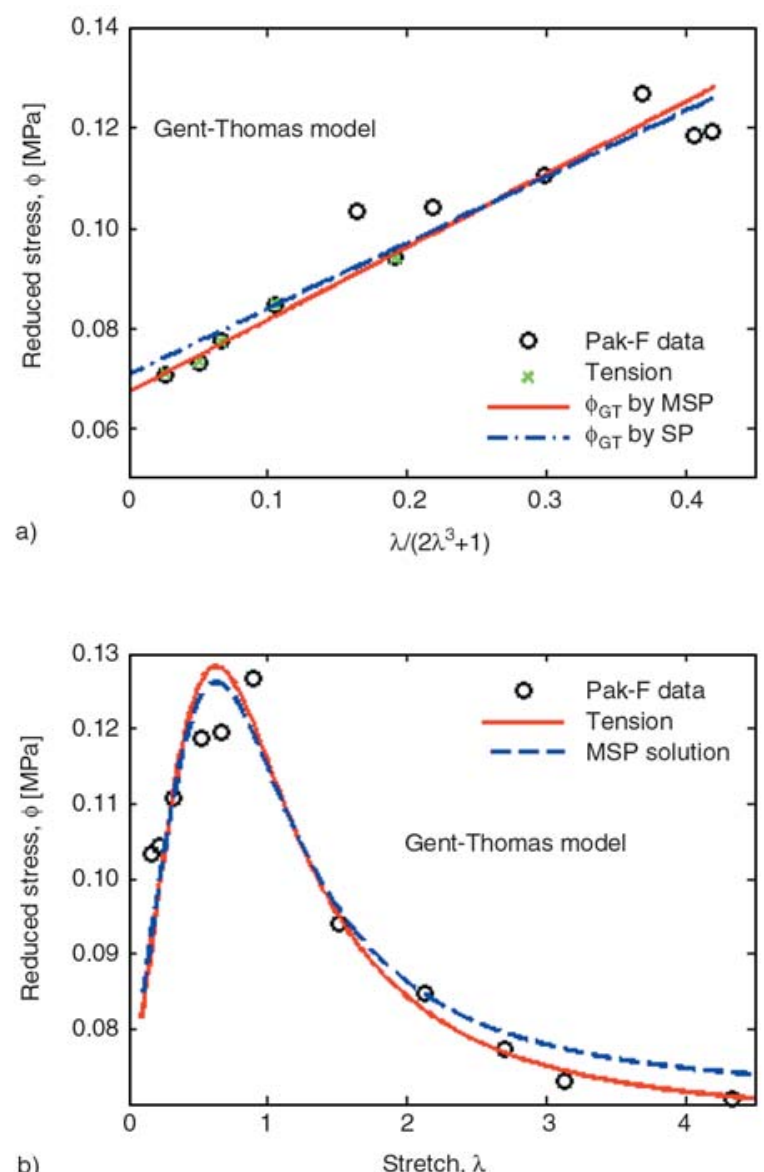

From Equations (3) and (5), a change of variable is not possible, because the change $X=\lambda /\left(2 \lambda^{3}+1\right)$ is not a univocal function. This necessitates the use of the graphical step-by-step approach [12]. As previously stated, when plotting $\phi(x)$ versus $\lambda /\left(2 \lambda^{3}+1\right)$, the domain where the curve is linear corresponds to the valid domain of the Gent-Thomas model, Figures $1 \mathrm{a}, 1 \mathrm{~b}$. The value of the parameter $K_{2}$ is given by the gradient of the straight line and that of $K_{1}$ is the intercept. These parameters can be graphically estimated or, for more accuracy, can be evaluated by the least squares method within the appropriate domain [13] identified (within the linear curve area).

\subsection{Results}

Let us consider for illustration purposes: on one hand, data of uniaxial tension of the Pak-Flory elastomer [14], Figure 1; and on the other hand, those of the Treloar rubber [15], Figure 2.

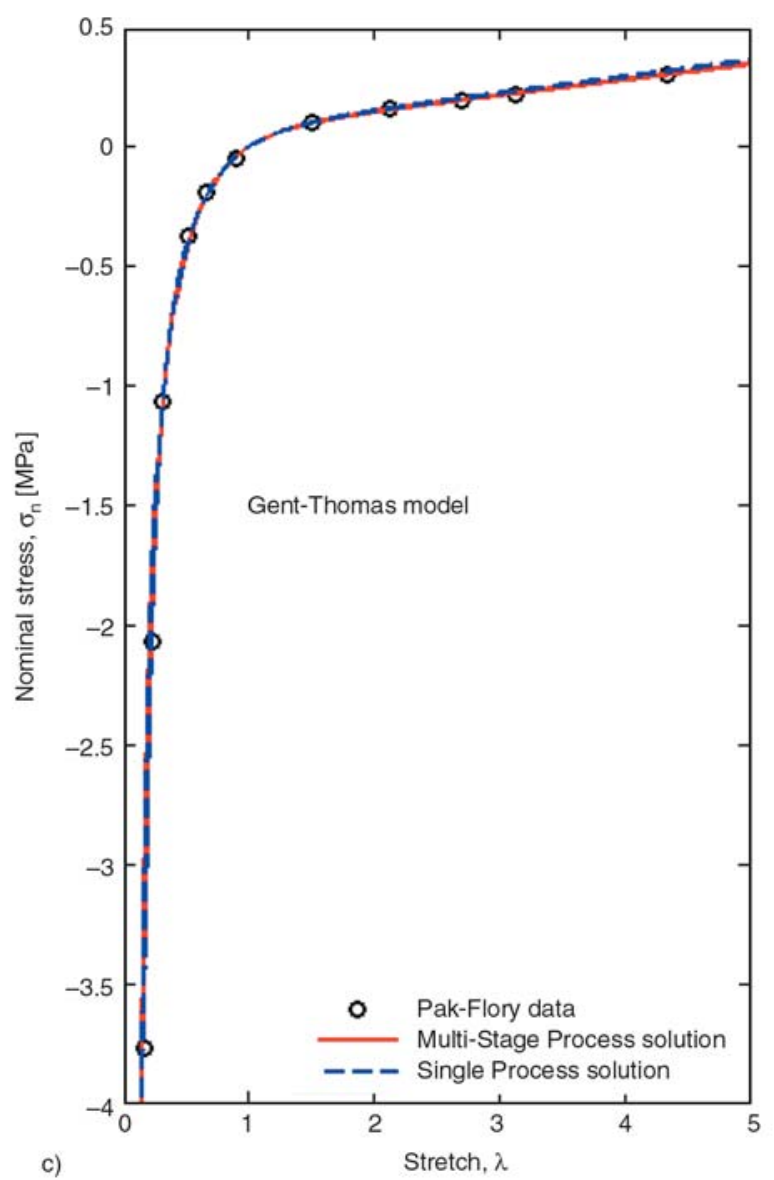

Figure 1. Validating and fitting the Gent-Thomas model to Pak-Flory elastomer data. (a): validating domain of interest and parameters evaluation (Straight line); (b): estimated reduced curves compared to data; (c): estimated compression-extension curves compared to data. 

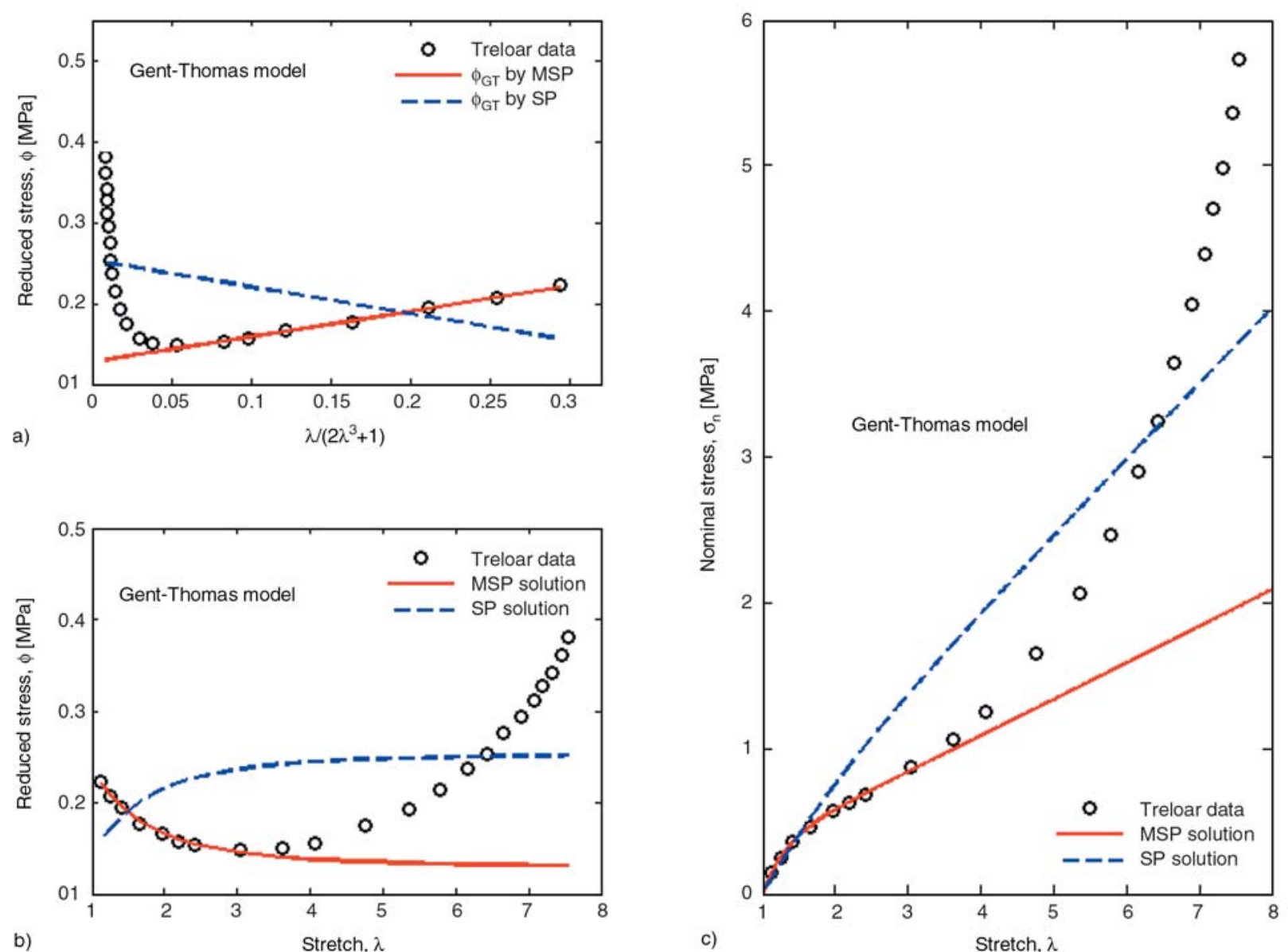

b)

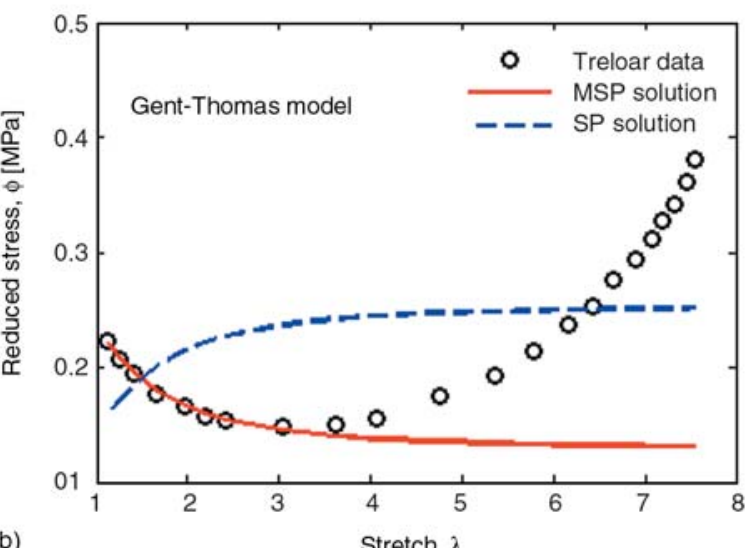

c)

Figure 2. Validating and fitting the Gent-Thomas model to Treloar rubber data. (a): validating domain of interest and parameters evaluation (Straight line); (b): estimated reduced curves compared to data; (c): estimated compression-extension curves compared to data.

- For the Pak-Flory data, one can use the single as well as the multi-stage procedure. The multi-stage procedure (where only extension data are taken into account for the resolution) permits just more precision on the estimated parameter values, Figure 1a. The parameter values obtained are recorded in Table 1. Figure 1b, $1 \mathrm{c}$ shows a good fit of data from compression to moderate tension, by the single as well as the multi-stage process. The Gent-Thomas model is valid over the entire experimental deformation domain of Pak-Flory, i.e., moderate and transition deformation zones. For this band of deformation, the use of the ordinary (single process) least squares procedure remains operational for validating and fitting the Gent-Thomas model to data. - For the Treloar data, one sees in Table 2 that the coefficient $K_{2}$ is negative-valued. This is at variance with the regression analysis of the constrained energy function by Johnson et al. [16]. From Figure $2 \mathrm{a}$, we see that the single process is irrelevant. Figure $2 b, 2 c$ shows that the solution curves diverge markedly from the whole experimental data by the single process. While by the multi-stage process, a good fit of data is observed from small to moderate deformations though a noticeable deviation appears at large deformations. This is understandable because one does not a priori know the domain where the restricted Gent-Thomas model is suitable. The ordinary procedure uses the whole experimental data to evaluate the energy parameters in a single process. Considering a single process means that the reduced stress should be linear in the whole deformation region when plotting $\phi(\lambda)$ versus the generating function $\xi_{2}(\lambda)$. This is not always accurate since experimental data do not corroborate it in Figure 2a. This is due to the fact that the Treloar experimental domain is complete: moderate deformation field, transition zone and stiffening domain. This shows that the Gent-Thomas model is only valid in a limited deformation region, i.e., stretches under the upturn point of the reduced stress curve (in other words, at the falling part of the curve), 
Table 2. Estimated parameters of the Gent-Thomas model from Treloar rubber data

\begin{tabular}{|l|c|c|}
\hline \multicolumn{1}{|c|}{ Parameters } & $\mathbf{K}_{\mathbf{1}}$ & $\mathbf{K}_{\mathbf{2}}$ \\
\hline Simple Process (SP) & 0.2543 & -0.3296 \\
\hline Multi-Stage Process (MSP) & 0.1286 & 0.3137 \\
\hline
\end{tabular}

Figure 2b. Consequently, it is a partial model, which is applicable and functional in a limiting area, within a restricted deformation domain of interest located under the inflexion point of the tension curve, Figure 2c. Table 2 presents estimated parameters by the multi-stage process compared to those by the single process. Thus, by the multistage process, one first of all looks for the valid domain of a model before evaluating relative parameters. For a rubber material, if one considers just two deformation zones: the first zone, where the tension curve is concave, as moderate stretches; the other zone, where it is rather convex, as the high deformations region, one can deduce that the GentThomas model is suitable only for moderate stretches, i.e., under the stiffening deformation zone, Figure 2c.

\section{Simulation: Validating domain and fitting the molecular Flory-Erman model}

It is well known that for a constrained chain model, parameters are obtained from molecular consideration. But we propose a simulation approach for estimating these parameters by means of a numerical approach in order to appreciate the efficiency of the studied method.

\subsection{Uniaxial behavior law}

The constrained chain model of Erman-Mannorie $[17,18]$ is given by Equation (17):

$$
\begin{aligned}
W_{E M}= & \frac{1}{2} \eta k T \sum_{j=1}^{3}\left(\lambda_{j}^{2}-1\right)+ \\
& \frac{1}{2} \eta k T \sum_{j=1}^{3}\left(\frac{N}{\eta}\left[B_{j}+D_{j}-\ln \left(1+B_{j}\right)-\ln \left(1+D_{j}\right)\right]\right)
\end{aligned}
$$

where $B_{j}, D_{j}$ and $h(\lambda)$ are given by Equations (18), (19) and (20):

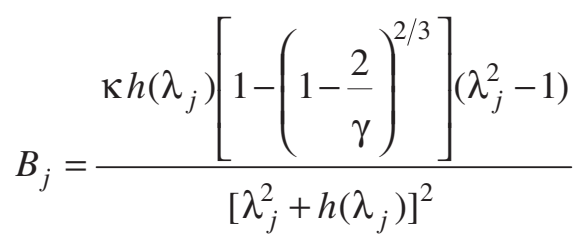

$D_{j}=\frac{\lambda_{j}^{2} B_{j}}{h\left(\lambda_{j}\right)}$

$h\left(\lambda_{j}\right)=\kappa\left[1+\frac{\left(1-\frac{2}{\gamma}\right)^{2 / 3}\left(\lambda_{j}^{2}-1\right)}{3}\right]$

$\eta$ is the cyclic rank of a network, $k$ the Boltzmann's constant, $T$ the absolute temperature, $\gamma$ is the number of chains meeting at a junction (the functionality of the network junction), $\kappa$ is a measure of strengths of the constraints, $N$ is the chain density ( $\eta=N / 2$ for a perfect tetra functional network).

For the Flory-Erman model $[18,19] \gamma$ is equal to two, so $h(\lambda), B_{j}$ and $D_{j}$ are given by Equations (21), (22) and (23):

$h\left(\lambda_{j}\right)=\kappa$

$B_{j}=\kappa^{2} \frac{\lambda_{j}^{2}-1}{\left(\lambda_{j}^{2}+\kappa\right)^{2}}$

$D_{j}=\kappa \lambda_{j}^{2} \frac{\lambda_{j}^{2}-1}{\left(\lambda_{j}^{2}+\kappa\right)^{2}}$

From Equation (1), the behavior law is given by Equation (24) $\left(\lambda_{1}=\lambda ; \lambda_{2}=\lambda_{3}=\lambda^{-1} ; \eta=N / 2\right)$ :

$$
\begin{aligned}
& \sigma_{n}=\frac{N k T}{2}\left(\lambda-\frac{1}{\lambda^{2}}\right)+ \\
& \frac{N k T}{2} \sum_{j=1}^{3}\left(\frac{\partial B_{j}}{\partial \lambda}+\frac{\partial D_{j}}{\partial \lambda}-\frac{1}{1+B_{j}} \frac{\partial B_{j}}{\partial \lambda}-\frac{1}{1+D_{j}} \frac{\partial D_{j}}{\partial \lambda}\right)
\end{aligned}
$$

This expression shows a unique corresponding generating function $\xi_{1}(\lambda)$.

Let us consider $\xi_{1}(\lambda)=\xi_{F E}(\lambda)$. Hence, $\xi_{F E}(\lambda)$ is given by Equation (25): 


$$
\begin{aligned}
\xi_{F E}(\lambda) & =\lambda-\frac{1}{\lambda^{2}}+ \\
\sum_{j=1}^{3} & \left(\frac{\partial B_{j}}{\partial \lambda}+\frac{\partial D_{j}}{\partial \lambda}-\frac{1}{1+B_{j}} \frac{\partial B_{j}}{\partial \lambda}-\frac{1}{1+D_{j}} \frac{\partial D_{j}}{\partial \lambda}\right)
\end{aligned}
$$

\subsection{Ordinary procedure for parameters identification}

For the ordinary procedure, the base of approximation contains a unique generating function and is given by Equation (26):

$$
\langle P(\lambda)\rangle=\left\langle\xi_{F E}(\lambda)\right\rangle
$$

As a result, the parameter $N k T$ is given by Equation (27):

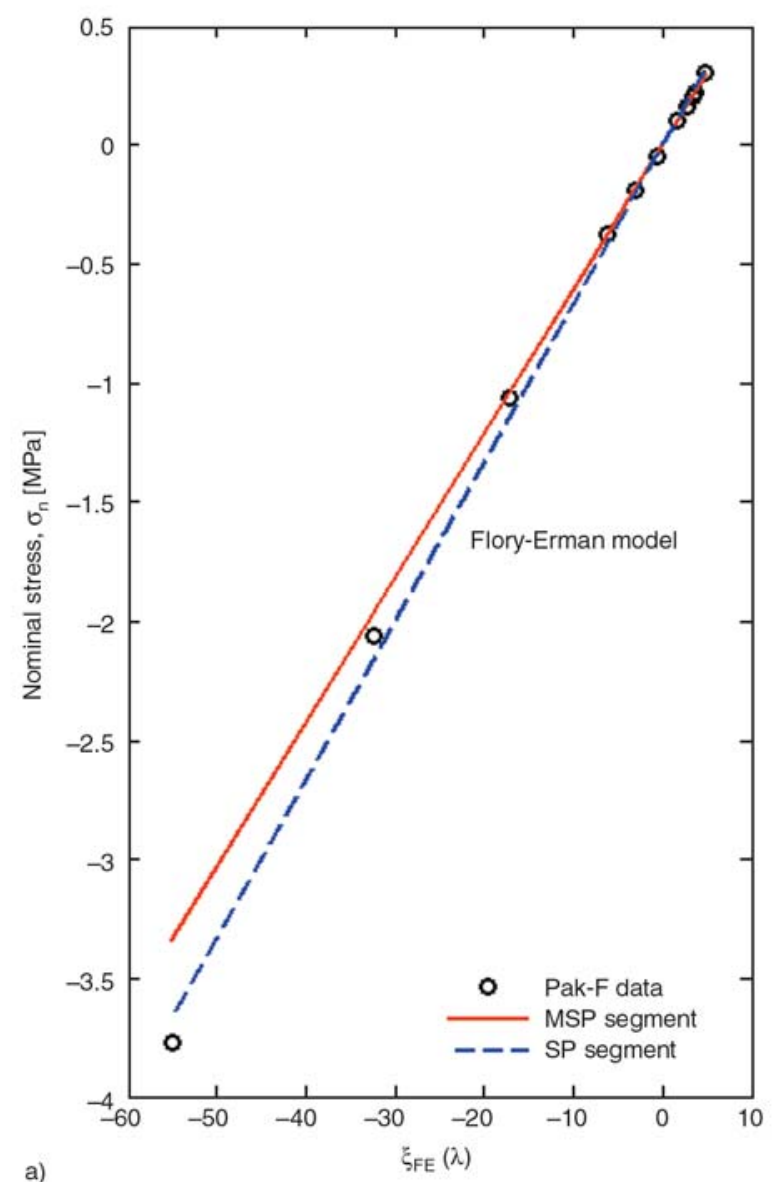

$$
N k T=\frac{\sum_{k=1}^{M} \xi_{F E}\left(\lambda_{k}\right) \sigma_{n k}}{\sum_{k=1}^{M} \xi_{F E}^{2}\left(\lambda_{k}\right)}
$$

where $\left(\lambda_{k}, \sigma_{n k}\right)$ are experimental data. The estimated parameters of the Flory-Erman model are recorded in Tables 3 and 4 and are compared to theoretical values (real values, from molecular considerations) $[19,20]$. Figure 3 depicts the model prediction in comparison with Pak-Flory data. From this Figure, it emerges that the usual single procedure can be applied in this case; while in Figure 4 , one sees that the single process is incongruous, the reasons are enumerated above.

Table 3. Estimated parameter $N k T$ for the Flory-Erman model from Pak-Flory elastomer data $(\kappa=3.58)$

\begin{tabular}{|l|c|c|}
\hline \multicolumn{1}{|c|}{ Parameter NkT } & $\begin{array}{c}\text { Estimated } \\
\text { value }\end{array}$ & $\begin{array}{c}\text { Theoretical value [20] } \\
\text { (Real value) }\end{array}$ \\
\hline Simple Process (SP) & 0.0660 & 0.06269 \\
\hline Multi-Stage Process (MSP) & 0.0608 & - \\
\hline
\end{tabular}

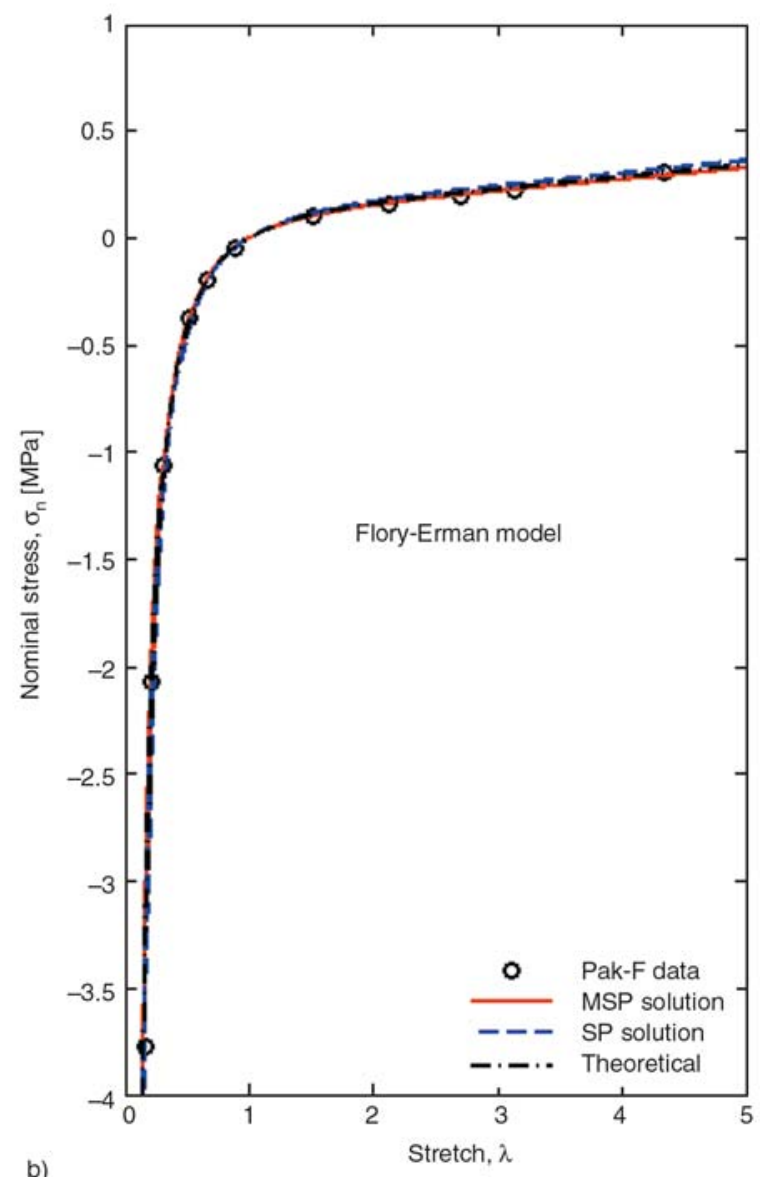

b)

Figure 3. Validating and fitting the Flory-Erman model to Pak-Flory data. (a): validating domain of interest and parameters evaluation; (b): estimated compression-extension curves compared to data. 

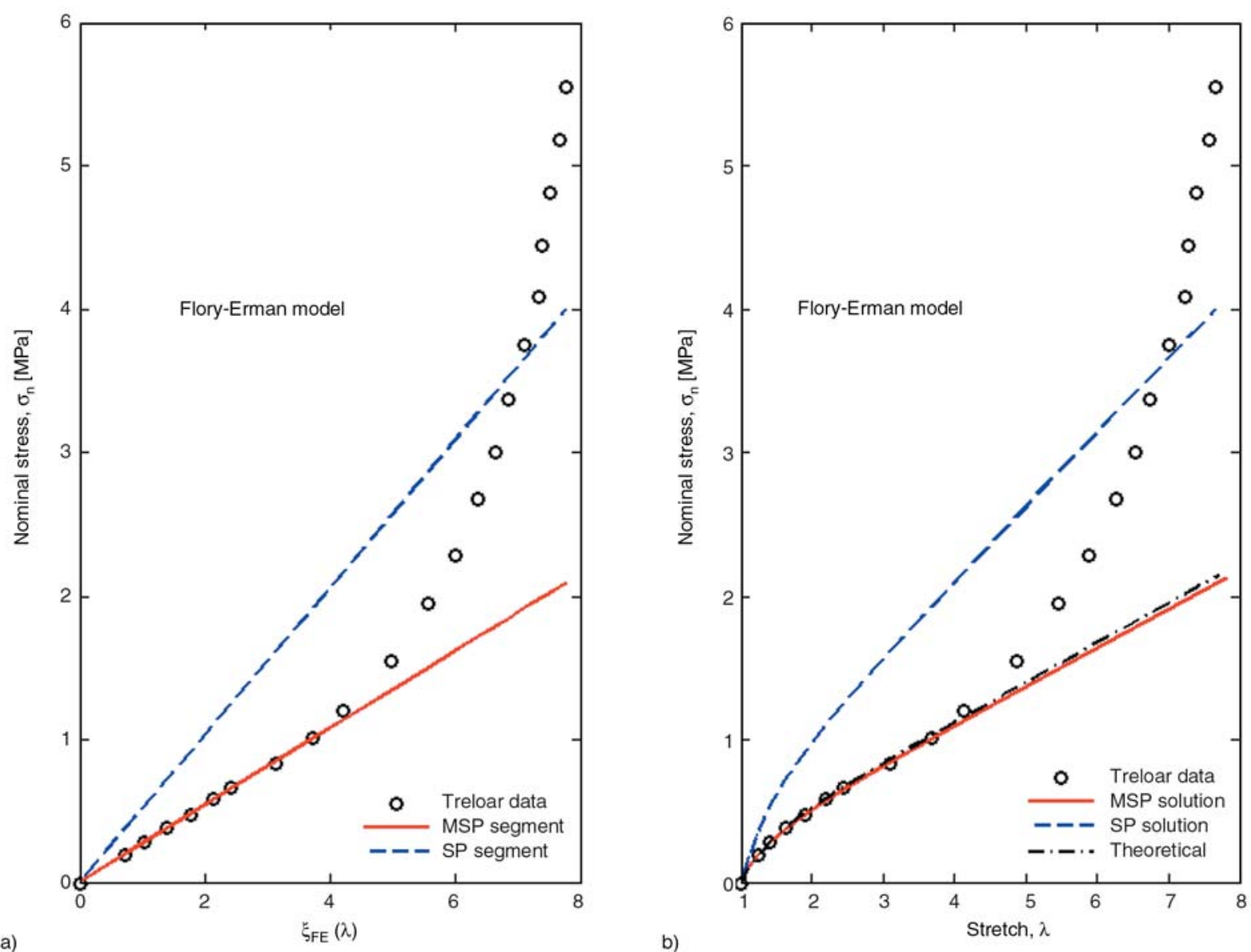

Figure 4. Validating and fitting the Flory-Erman model to Treloar data. (a): validating domain of interest and parameters evaluation; (b): estimated compression-extension curves compared to data.

Table 4. Estimated parameter $N k T$ for the Flory-Erman model from Treloar rubber data $(\kappa=0.68)$

\begin{tabular}{|l|c|c|}
\hline \multicolumn{1}{|c|}{ Parameters NkT } & $\begin{array}{c}\text { Estimated } \\
\text { value }\end{array}$ & $\begin{array}{c}\text { Theoretical value [19] } \\
\text { (Real value) }\end{array}$ \\
\hline Simple Process (SP) & 0.5151 & 0.55 \\
\hline Multi-Stage Process (MSP) & 0.5382 & - \\
\hline
\end{tabular}

\subsection{Multi-stage procedure for identification}

As previously seen, to evaluate parameters by the multi-stage procedure, one plots the reduced stress versus the generating function $\xi_{F E}(\lambda)$. It permits both the determination of the valid domain of the model, i.e., where the curve is linear; and the value of the parameter $N k T$, which is given by the gradient of the straight part of the curve, Figures $3 \mathrm{a}$ and 4a. Tables 3 and 4 show the estimated and the theoretical parameter values of the Flory-Erman model. A comparison of theoretical and estimated predictions of the model with data is illustrated in Figure $3 b$ for Pak-Flory elastomer and in Figure $4 b$ for Treloar rubber.
Let us note that the Gent-Thomas and the FloryErman models give similar results [21].

\section{Complete models}

This paper presents how to validate a partial model in general and that of Gent-Thomas in particular, because of difficulties when using the ordinary identification procedure. The complete modeling is not considered in this work.

Let us note that for a complete model such as that of Pucci-Saccomandi (also called Gent-Gent model) [22] given by Equation (28) or that of Beda (the extended Gent-Thomas model) [21] given by Equation (29), the ordinary as well as the multistage procedure remains appropriate for evaluating the parameters $[9,12,13,21]$. The Gent-Gent model is given by Equation (28):

$$
W_{P S}=K_{2} \ln \frac{I_{2}}{3}-\frac{\mu}{2} J_{m} \ln \left(1-\frac{I_{1}-3}{J_{m}}\right)
$$


where $J_{m}=I_{m}-3 ; K_{2}$ and $\mu$ are material parameters and $I_{m}$ is the limiting value of the invariant $I_{1}$ to the deformation when the network is fully stretched.

The Beda (extended Gent-Thomas) model is given by Equation (29):

$$
\begin{aligned}
W_{B}= & K_{1}\left(I_{1}-3\right)+K_{2} \ln \frac{I_{2}}{3}+ \\
& K_{3}\left(I_{1}-3\right)^{M-1}+K_{4}\left(I_{1}-3\right)^{M}
\end{aligned}
$$

where $K_{i}$ are positive real numbers characteristic of material. The integer $M$ (condition apply: $M \geq 3$ ) is the order of the model or material order [21].

Let us also note that the Pucci-Saccomandi (GentGent) and Beda (extended Gent-Thomas) models give similar precise results [21].

\section{Conclusions}

A technique of validating partial hyperelastic models over a complete experimental range of deformation is set out. The Gent-Thomas model, an earlier and starting partial model, which successfully verifies experimental and theoretical requirements, but unfortunately not sufficiently exploited in hyperelastic modeling, can now be easily employed in rubber modeling and characterization. It comes out that the Gent-Thomas model is valid only under the stiffening deformation domain of rubber material.

\section{References}

[1] Mooney M.: A theory of large elastic deformation. Journal of Applied Physics, 11, 582-592 (1940).

[2] Gent A. N., Thomas A. G.: Forms for the stored (strain) energy function for vulcanized rubber. Journal of Polymer Science, 28, 625-637 (1958).

[3] Valanis R. C., Landel R. F.: The strain-energy function of a hyperelastic material in terms of the extension ratios. Journal of Applied Physics, 38, 29973002 (1967).

[4] James A. G., Green A.: Strain energy function of rubber. II. The characterization of filled vulcanizates. Journal of Applied Polymer Science, 19, 2319-2330 (1975).

[5] Ogden R. W.: Non-linear elastic deformations. Ellis Harwood, Chichester (1984).

[6] Lin D. C., Dimitriadis E. K., Horkay F.: Elasticity of rubber-like materials measured by AFM nanoindentation. Express Polymer Letters, 1, 576-584 (2007).
[7] Yeoh O. H., Fleming P. D.: A new attempt to reconcile the statistical and phenomenological theories of rubber elasticity. Journal of Polymer Science, Part B: Polymer Physics, 35, 1919-1931 (1997).

[8] Beda T.: Reconciling the fundamental phenomenological expression of the strain energy of rubber with established experimental facts. Journal of Polymer Science, Part B: Physics, 43, 125-134 (2005).

[9] Ogden R. W., Saccomandi G., Sgura I.: Fitting hyperelastic models to experimental data. Computational Mechanics, 34, 484-502 (2004).

[10] Rivlin R. S., Saunders D. W.: Large elastic deformation of isotropic materials. VII. Experiments of the deformation of rubber. Philosophical Transaction of the Royal Society, Series A: Mathematical and Physical Sciences, 243, 251-288 (1951).

[11] Rivlin R. S.: Large elastic deformations. in 'Rheology: Theory and Applications' (ed.: Eirich F. R.) Academic Press, New York, 351-385 (1956).

[12] Beda T., Chevalier Y.: Non-linear approximation method by an approach in stages. Computational Mechanics, 32, 177-184 (2003).

[13] Beda T.: Combining approach in stages with least squares for fits of data in hyperelasticity. Comptes Rendus Mecanique, 334, 628-633 (2006).

[14] Pak H., Flory P. J.: Relationship of stress to uniaxial strain in crosslinked poly(dimethylsiloxane) over the full range from large compressions to high elongations. Journal of Polymer Science, Polymer Physics Edition, 17, 1845-1854 (1979).

[15] Treloar L. R. G.: The physics of rubber elasticity. Oxford University Press, Oxford (1975).

[16] Johnson A. R., Quigley C. J., Mead J. L.: Large strain viscoelastic constitutive models for rubber, Part I: Formulations. Rubber Chemistry and Technology, 67, 904-917 (1994).

[17] Erman B., Monnerie L.: Theory of elasticity of amorphous networks: Effect of constrains along chains. Macromolecules, 22, 3342-3348 (1989). Correction: 25, 4456 (1992).

[18] Flory P. J., Erman B.: Theory of elasticity of polymer networks, 3. Macromolecules, 15, 800-806 (1982).

[19] Boyce M. C., Arruda E. M.: Constitutive models of rubber elasticity: A review. Rubber Chemistry and Technology, 73, 504-523 (2000).

[20] Han W. H., Horkay F., McKenna G. B.: Mechanical and swelling behaviors of rubber: A comparison of some molecular models with experiment. Mathematics and Mechanics of Solids, 4, 139-167 (1999).

[21] Beda T.: Modeling hyperelastic behavior of rubber: A novel invariant-based and a review of constitutive models. Journal of Polymer Science, Part B: Physics, 45, 1713-1732 (2007).

[22] Pucci E., Saccomandi G.: A note on the Gent model for rubber-like materials. Rubber Chemistry and Technology, 75, 839-851 (2002). 